\title{
Methods for estimating moment of inertia of cricket bats
}

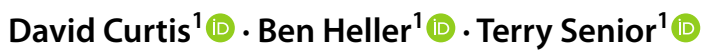

Accepted: 28 April 2021 / Published online: 8 May 2021

(c) The Author(s) 2021

\begin{abstract}
Mass moment of inertia is a key inertial property of cricket bats and should be used in selection to optimise performance. Players currently rely on a subjective assessment of how the bat feels when swung supported only by a value for bat mass from the manufacturer. This reliance on a subjective assessment is because the moment of inertia of a bat typically requires a pendulum method to measure with sufficient accuracy. In this study, two methods for estimating moment of inertia were tested. The hypotheses were that (1) an acceptable estimate of moment of inertia could be calculated using a beam model approach, and (2) the inertial property first moment could act as a proxy measure for moment of inertia. Experimental values for moment of inertia were obtained using a pendulum method. The two-section beam model showed an error of $0.43-0.53 \%$ between model and experimental values based on a Root Mean Square Error of $0.0017 \mathrm{~kg} \mathrm{~m}^{2}$. First moment data were generated on 5005 bats spread across eight bat shapes. A correlation was shown between the measured value of first moment and the beam model value of moment of inertia, with an $R^{2}$ value $>0.992$ for all bat shapes. This study showed that a two-section beam model and first moment method for estimating cricket bat moment of inertia could be used to improve bat selection.
\end{abstract}

Keywords Cricket bat $\cdot$ Mass moment of inertia $\cdot$ Modelling $\cdot$ Centre of mass $\cdot$ Equipment selection

\section{Introduction}

The ability of players to swing an implement with optimum speed and accuracy is a key element of performance in many sports. While players have some control over the material performance of the implement through selection decisions based on perceived quality and cost, they have greater control on the selection of physical dimensions, albeit ones often constrained by rules applied by the sport's governing body. The implement's material densities and dimensions combine to determine its mass moment of inertia (MOI), which is the inertial property that describes its resistance to angular acceleration. There are three MOIs that act about the principle axes $(x, y, z)$ through the implement centre of mass (Fig. 1a). These MOIs were described for tennis rackets by Brody [1], later defined by Taraborelli et al. [2] as Transverse $(x)$, Polar $(y)$, and Lateral $(z)$, and are shown in Fig. $1 \mathrm{~b}$ for cricket bats. Application to performance of

David Curtis

d.curtis@shu.ac.uk

1 Sports Engineering Research Group, Advanced Wellbeing Research Centre, Sheffield Hallam University, Olympic Legacy Park, 2 Old Hall Road, Sheffield S9 3TU, UK cricket bats has been described by Eftaxiopoulou [3] and in cricket batting by Headrick et al. [4]. In cricket, the Transverse MOI about the handle is of most interest as this has the biggest effect on the swing of the bat in its predominant mode of use (Fig. 1b).

There is published research involving implement swing in baseball, golf, and tennis, with investigations on swing speed, MOI, and player performance [5-7]. Brody [1] was the first to describe the relevance of tennis racket MOI to players and demonstrated a method of measurement for Transverse $(x)$ and polar $(y)$ moments. For baseball, Fleisig et al. [8] showed that bat swing speed decreased as MOI increased. Cross \& Nathan [9] went on to show that the 'intrinsic power' of a tennis racquet, golf club and baseball bat correlates well with MOI but poorly with mass. Intrinsic power is a colloquial term coined by Cross \& Nathan to describe the implement's apparent coefficient of restitution, which determines the ball rebound speed. More recently, Schorah et al. [10] showed through a meta-analysis across several sports that swing acceleration correlates better with MOI than velocity, in accordance with the governing physics.

The cricket bat provides an interesting case study due to the wide range of inertial properties apparent in consumer 
Fig. 1 a cricket bat profile, $\mathbf{b}$ simplified schematic bat, and $\mathbf{c}$ equivalent unequal two-section one-dimensional beam

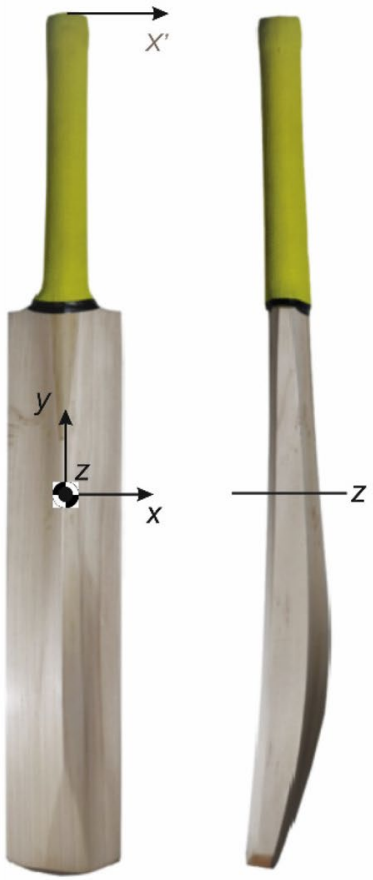

(a)

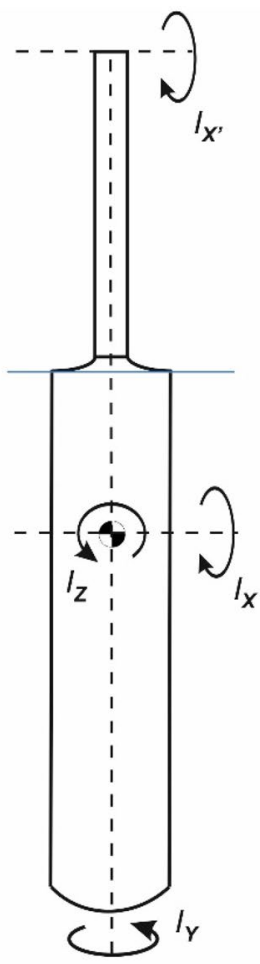

(b)

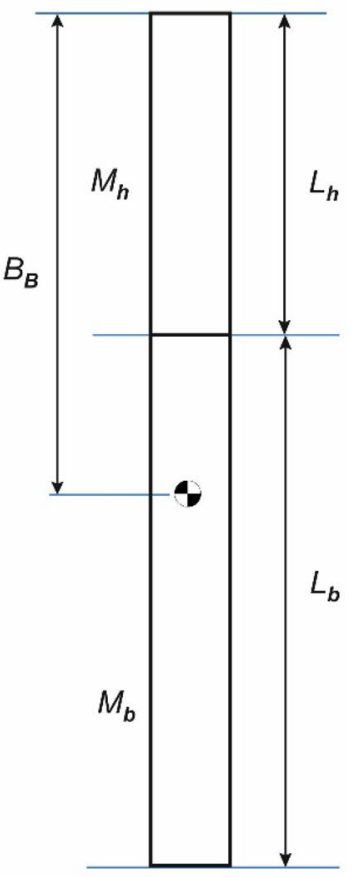

(c) products. The quality and performance of the bat materials aside, a functional bat is one that the player can manoeuvre at a range of speeds to make controlled strikes in defence or in attack for run scoring [11]. In a study on the factors affecting cricket batting performance, Peploe et al. [12] concluded that to maximise post-impact ball speed players should focus on striking it with the highest possible bat speed. The bat should also have an effective mass that enables maximum batted ball speed through the momentum transfer at impact. Cross \& Nathan [9] described the 'intrinsic power' of an implement being dependent on the effective mass at the point of impact. Therefore, finding a bat MOI that satisfies a player's specific requirements for bat speed and effective mass is an essential driver of performance.

MOI is not used in cricket as a parameter for bat selection, and there are two main limiting factors; (1) MOI is not provided by the manufacturers, and (2) there are no facilities at the point of purchase to measure and assess swing performance. Instead, current practice uses the knowledge of the bat's mass in conjunction with a subjective assessment of how the bat feels when swung, which is often referred to as 'pick-up' (e.g. heavy, light, balanced, toe-heavy). Similar to other sports implements, MOI is problematic in its practical measurement by cricket bat makers and players due to the complex three-dimensional geometry of the bat. Measurement currently requires the use of specialist equipment to implement a simple or bifilar pendulum test as described for tennis rackets by Brody [1], for baseball bats by Koenig [13], for cricket by Eftaxiopoulou [3] and the ASTM [14]. The process of measurement using this method is also timeconsuming in the context of production, which exerts a constraint on adoption as common practice.

An alternative to measuring MOI is to make a one-dimensional beam model of the cricket bat that closely matches more easily measured properties, such as mass, and centre of mass (CoM) location. The beam model has been demonstrated as a good estimator of MOI for tennis rackets, firstly by Cross [15], then refined by Goodwill [16], and recently validated for over 400 tennis rackets by Taraborrelli et al. [2]. Cross demonstrated the effectiveness of a simple twosection beam model, where the handle and frame were each represented by a one-dimensional beam of identical length but different mass. The limitation of uniform length and one beam to represent the frame was addressed by Goodwill who developed a one-dimensional five-section beam model that better represented the mass distribution of the frame. This five-section beam model produced identical mass and CoM location against seven test rackets, and MOI values within $2 \%$. Allen et al. [17], working from Goodwill, tested unequal two-section and five-section models on 100 rackets. Model MOI values produced a root mean square error (RMSE) of $0.0015 \mathrm{~kg} \mathrm{~m}^{2}$ for both models ( $3 \%$ of a typical racket MOI). Thus indicating that the unequal two-section beam model is comparable to a five-section beam model, yet simpler to 
implement. Taraborelli et al. [2] further demonstrated the effectiveness of the unequal two-section model by testing over 400 diverse rackets, showing model values with RMSE of $0.0013 \mathrm{~kg} \mathrm{~m}^{2}$.

It is proposed that predicting MOI with beam models can apply in cricket bats. If the model is more accurate than player sensitivity to MOI changes, then this offers a method for rapid calculation since the model only requires the measurement of mass, CoM location, and section lengths as inputs. Kreifeldt and Chuang [18] studied human sensitivity to MOI. Ten males and ten females swung a hollow tube with concealed masses, with the tube dimensions set to simulate a tennis racket. Tester experience was not described, so it is unknown if they were sports players. The authors commented that the infrequent need to interact with angular accelerations prevented reliable comparisons of MOI, on the basis that it was an unrecognisable property in how an object felt when swung. However, in ball sports using swung implements the players are interacting frequently with this phenomenon. A study by Brody [19] on MOI sensitivity in tennis rackets demonstrated that non-tennis players required over 25\% MOI change to detect a difference. Proficient tennis players required over $2.5 \% \mathrm{MOI}$ change to detect a difference. The work of Brody therefore provides an initial basis for assessing the efficacy of a beam model for estimating MOI of cricket bats.

Another approach to estimating MOI is to use a first mass moment as a proxy. First mass moment is the product of mass and the distance of the centre of mass from a reference position. In golf, first moment is referred to as swingweight [20]. A reference position of $0.356 \mathrm{~m}$ (14 in) from the handle end of the golf club was established in the 1920s by Robert Adams and termed the Lorythmic Scale [21]. The Lorythmic Scale allowed a set of golf club irons to be matched on the property of swingweight. Cross and Nathan [9] showed that a set of irons could also be matched for MOI to within $0.15 \%$ if the reference position was changed to $0.47 \mathrm{~m}$ from the handle end. The implication is that first moment at a re-defined reference position could be a proxy for MOI. Harper et al. [22] showed that golfers were unable to perceive differences of $< \pm 3$ points in golf club swingweight (first moment) on the Lorythmic scale. Three Lorythmic swingweight points is equal to $0.00432 \mathrm{~kg} \mathrm{~m}$. With a typical swingweight for a golf iron at $0.155 \mathrm{~kg} \mathrm{~m}$ [9], three swingweight points equates to under $3 \%$ of the total swingweight. This indicates that the ability to match MOI on a set of irons to within $0.15 \%$ is well below the limit of a player's perception of swingweight changes. Through the calculation of Weber fractions (a measure of differential sensitivity to a sensory stimulus around a reference value), Kreifeldt and Chuang [18] also noted that values of Weber fraction did not differ widely when comparing test objects with mass-MOI ratios of 1000:1 and 10:1. This finding indicates that sensitivity of golfers to first mass moment changes found by Harper could be similar to cricket bats whose mass-MOI ratio (typically 3.3:1, see Table 1) is of the same magnitude as a golf iron (typically 1.5:1 [9]). This suggests that first mass moment could be used as a suitable proxy for MOI in cricket bats. Therefore, this study developed and tested two methods of estimating MOI for cricket bats using (i) a onedimensional beam model, and (ii) first moment as a proxy.

\section{Methods}

\subsection{Experimental mass moment of inertia}

The MOI acting about the handle end $\left(I_{x^{\prime}}\right)$ of the cricket bat, as shown in Fig. 1b, was determined by turning the bat into a physical pendulum. The pendulum method using a cylindrical pivot adopted in this study has been shown to measure MOI of rods to within $2 \%$ of theoretical values by Spurr et al. [23], and Allen et al. [17]. Here, the pivot position was taken at the handle end. Other studies [1, 2, 14, 24] have used a pivot between 7.5 and $15 \mathrm{~cm}$ from the handle end, either with a rod passed through a drilled hole in the handle, or a clamping mechanism. The rationale is that these positions supposedly represent the pivot point when held by the hands of the player. Here, the handle end was chosen in the main to avoid damage to the handle, but has also been used in MOI studies using rods [5, 25]. The parallel axes theorem can be applied to translate the MOI to a different position, but it was not applied here.

A $25 \mathrm{~mm}$ diameter steel disc welded to a cylindrical steel rod of $6 \mathrm{~mm}$ diameter and $217 \mathrm{~mm}$ length was attached to the handle end by two small screws. The additional mass was $83.8 \mathrm{~g}$ and the estimated MOI of the disc-rod was $9.4 \times 10^{-6} \mathrm{~kg} \mathrm{~m}^{2}$, which is $<0.003 \%$ of the expected MOI of the bat $\left(0.3-0.4 \mathrm{~kg} \mathrm{~m}^{2}\right)$ and hence negligible. The disc-rod pivot was placed across two aluminium corner sections to act as 'knife edges' fixed to a frame to allow the bat to swing freely. Each bat was displaced from the vertical position by up to $10^{\circ}$ to ensure the small angle approximation $\sin a \approx a$ (for $a \leq 14^{\circ}$ ) was valid according to the principles of reducing the system to a pendulum with simple harmonic motion. The time for thirty full swing cycles was recorded using a stopwatch. Five repeat measures were taken for each bat to minimise manual timing errors, with the mean across the five measurements divided by the number of cycles to yield the period of the pendulum $T$, used for calculation of MOI in Eq. 1. The mass of each bat was measured to within $1 \mathrm{~g}$ using a Mettler PM16 digital scale. Location of the bat CoM was determined using the first moment measurement tool described in 2.2. MOI was then determined from: 
$I=T^{2} \frac{m g d}{4 \pi^{2}}$,

where $m$ is bat mass, $d$ is the distance between the pivot point (reference position) and the CoM. The order of magnitude of $I$ for full-size cricket bats covers a bat mass range of $1.0-1.3 \mathrm{~kg}$ as typically produced by cricket bat manufacturers. Pendulum measurements were carried out on 26 bats from four manufacturers.

\subsection{Experimental first moment}

Figure 2 shows a schematic of the bespoke device for measuring first moment. Two load cells (LCM Systems model SPB-8 $3 \mathrm{~kg}$ ) were fitted $0.753 \mathrm{~m}\left(d_{1}\right)$ apart on a horizontal plane inside an aluminium cradle, with an offset from the reference position (handle end) of $0.097 \mathrm{~m}\left(d_{2}\right)$. Load cell measurement accuracy was $< \pm 0.02 \%$ of the rated load, which on a representative $1.2 \mathrm{~kg}$ bat equates to $\pm 0.6 \mathrm{~g}$. An aluminium tray was located in the cradle to support the bat on the load cells. The tray provided a closed end that acted as the datum for the end of the bat handle to sit against to ensure $d_{2}$ was constant (shown in Supplementary Material 1). A small, printed circuit board (PCB) strain gauge amplifier conditioned the load cell output. A National Instruments digital acquisition unit (model NI-USB6009) converted the analogue signal from the strain gauge amplifier to a digital format for USB connection to a laptop. Bespoke operating software for the rig was created using Microsoft Visual Basic within.Net Framework 3.5, and output data were stored automatically in a Microsoft Access database. The system was calibrated using a $2 \mathrm{~kg}$ mass. The robust design enabled data collection in a cricket bat production facility.

Referring to Fig. 1 bat mass $M_{\mathrm{B}}=M_{1}+M_{2}$, CoM location $B_{\mathrm{B}}=d_{\mathrm{B}}+d_{2}$, and $d_{\mathrm{B}}=\left(M_{1} d_{1}\right) / M_{\mathrm{B}}$. First moment $\left(Q_{\mathrm{B}}\right)=M_{\mathrm{B}} \times B_{\mathrm{B}}$ and is therefore calculated as:
$Q_{\mathrm{B}}=\left(M_{1}+M_{2}\right)\left[\left(\frac{M_{1} d_{1}}{M_{\mathrm{B}}}\right)+d_{2}\right]$.

The measurement device was placed in the factory of a bat producer for a period of nine weeks to obtain the first moment on all bats produced in that period. This served the purpose of acquiring a large data set across eight bat shapes, with differing combinations of swell depth, edge size and volume. Images for the bat shapes are provided in Supplementary Material 2. Additional calculations were performed on the first moment data obtained from the device to generate an estimate of MOI using the two-section beam model. Bat handle and blade length measurements were added for this step.

\subsection{Two-section beam model}

The two-section one-dimensional beam equivalent for a cricket bat is similar to the tennis racket in that the bat is formed by a handle and a blade, just as a racket is formed by a handle and head. Three inertial mass properties were defined by $M_{\mathrm{B}}=M_{\mathrm{h}}+M_{\mathrm{b}}$, where $M_{\mathrm{B}}$ is the mass of the bat, $M_{\mathrm{h}}$ is the mass of the handle, and $M_{\mathrm{b}}$ and is the mass of the blade. The CoM location referenced to the handle end is derived by:

$B_{\mathrm{B}}=\frac{M_{\mathrm{h}}\left(\frac{L_{\mathrm{h}}}{2}\right)+M_{\mathrm{b}}\left(L_{\mathrm{h}}+\left(\frac{L_{\mathrm{b}}}{2}\right)\right)}{M_{\mathrm{B}}}$,

where, $L_{\mathrm{h}}$ is the length of the handle, and $L_{\mathrm{b}}$ is the length of the blade (Fig. 1c). Given that there is no obvious intersection of handle and blade that could be used as a reference point the interface datum for handle and blade was defined by a line drawn on the face of the bat between the corners of the shoulders of the blade (Fig. 1b). This is the least arbitrary position since it is the most consistent of measures across bats. For the curved toe of the blade the datum was taken as the end of the blade as measured through the centreline. A
Fig. 2 Schematic of the system function for calculating first moment

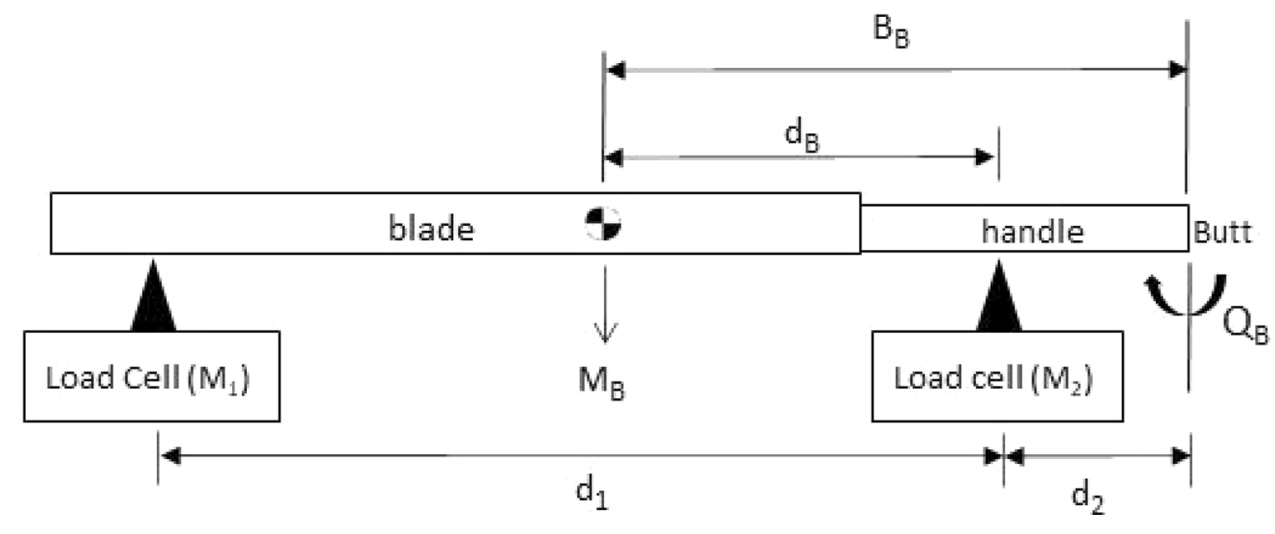


Table 1 Mass, geometric, and inertial bat properties (mean \pm standard deviation, and range)

\begin{tabular}{llllll}
\hline Bat mass $(\mathrm{kg})$ & COM location $(\mathrm{m})$ & Overall bat length $(\mathrm{m})$ & Handle length $(\mathrm{m})$ & Blade length $(\mathrm{m})^{\text {MOI }\left(\mathrm{kg} \mathrm{m}^{2}\right)}$ \\
\hline $1.170 \pm 0.0555$ & $0.500 \pm 0.006$ & $0.854 \pm 0.002$ & $0.301 \pm 0.003$ & $0.553 \pm 0.003$ & $0.3512 \pm 0.018$ \\
$1.068-1.265$ & $0.488-0.515$ & $0.850-0.857$ & $0.294-0.303$ & $0.550-0.563$ & $0.3209-0.3806$ \\
\hline
\end{tabular}

sensitivity analysis was performed on the datum position of the handle-blade and toe to assess magnitude of error from the manual measurement. The analysis showed a difference in MOI of less than $0.1 \%$ on $\pm 2 \mathrm{~mm}$ movement of this line.

The MOI for a uniform one-dimensional beam rotating about one end is given by:

$$
I_{x^{\prime}}=\frac{M_{\mathrm{B}} L_{\mathrm{B}}^{2}}{3} \text {. }
$$

For an unequal two-section one-dimensional beam this becomes:

$$
I_{x^{\prime}}=\frac{M_{\mathrm{h}} L_{\mathrm{h}}^{2}}{3}+\left(\frac{M_{\mathrm{b}} L_{\mathrm{b}}^{2}}{12}+M_{\mathrm{b}}\left(L_{\mathrm{h}}+\left(\frac{L_{\mathrm{b}}}{2}\right)\right)^{2}\right) .
$$

The bats measured were standard blade, and short handle. The study did not incorporate long handle, or long blade models, as these versions cover only a small percentage of the cricket bat supply. However, the method would also apply to these versions.

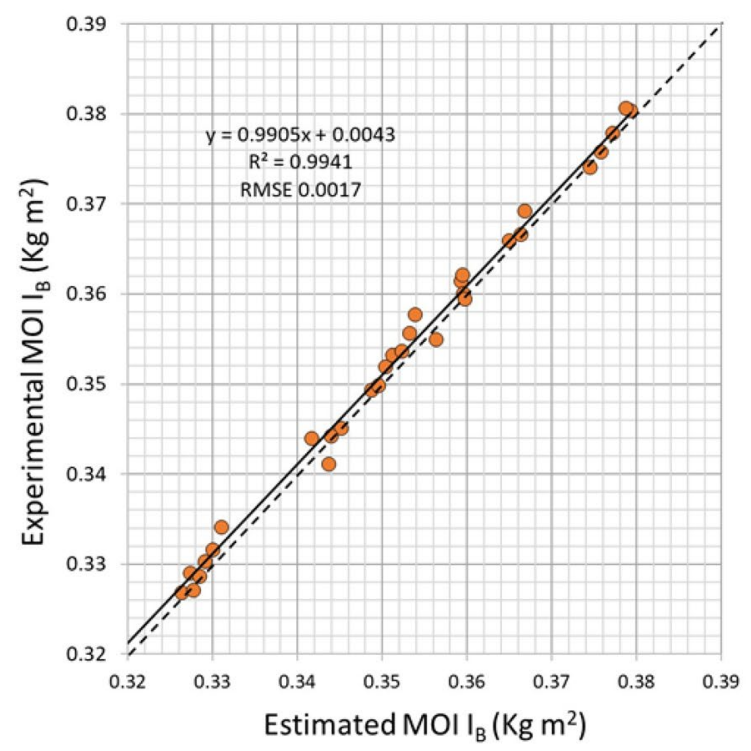

(a)

\section{Results}

\subsection{Experimental MOI}

Table 1 summarises the output from the pendulum measurements of 26 cricket bats from four manufacturers.

\subsection{Two-section beam model MOI}

Figure $3 \mathrm{a}$ shows the experimental values of $I_{\mathrm{x}^{\prime}}$ against the estimated $I_{\mathrm{x}^{\prime}}$ for the unequal two-section beam model. The output indicates that the model has a clear correlation with the experimental values, with a correlation coefficient $\left(R^{2}\right)$ of 0.994 , and RMSE of $0.0017 \mathrm{~kg} \mathrm{~m}^{2}$. Figure $3 \mathrm{~b}$ shows a Bland-Altman plot between experimental and estimated values. The mean difference (bias) between model and experiment across the sampled bats was $-0.001 \mathrm{~kg} \mathrm{~m}^{2}$, meaning the model tended to underestimate MOI when comparing to experimental values. Limits of agreement range from -0.0037 to $0.0017 \mathrm{~kg} \mathrm{~m}^{2}$, which represents a difference range of $-1.07-0.75 \%$ between model and experiment.

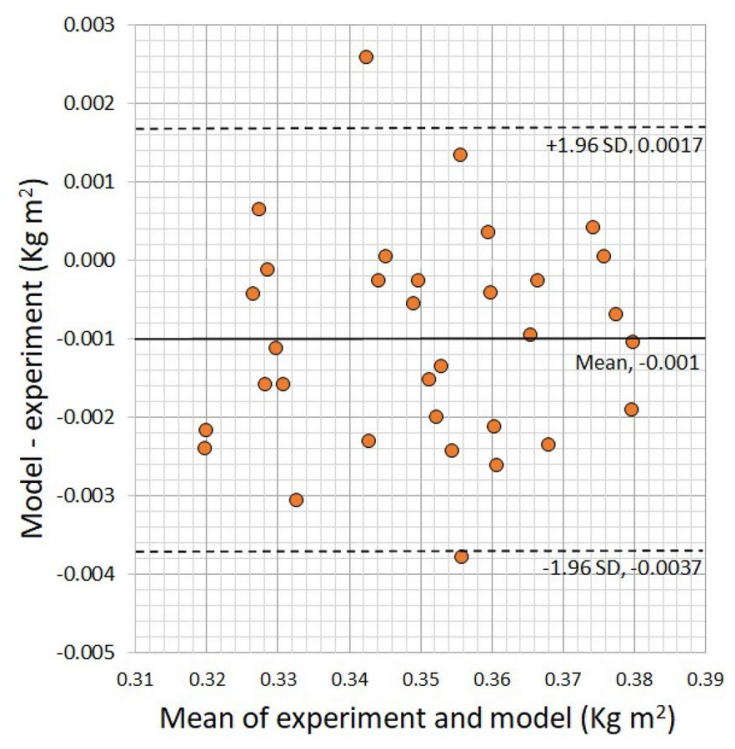

(b)

Fig. 3 a Two-section beam model estimated MOI and experimental MOI with best-fit line (solid) and exact-fit line (dashed), and b Bland-Altman plot, with mean difference (solid line) and limits of agreement (dashed) 


\subsection{Model versus first moment large data set comparisons}

Figure 4a shows measured first moment data against the twosection beam model MOI for one of the eight bat shapes measured in the factory (Bat 3, sample 1471 bats). This acted as a check on the accuracy of the two-section beam model. A regression analysis was conducted using the LINEST function in MS Excel to determine an estimate of uncertainty in the linear fit for Bat 3 data. The standard error for predicted MOI was $0.000859 \mathrm{~kg} \mathrm{~m}^{2}$, giving a $95 \%$ prediction interval of $\pm 0.0017 \mathrm{~kg} \mathrm{~m}^{2}$, which equates an estimated percentage error of $0.43-0.57 \%$ in the range of MOI plotted. Figure $4 \mathrm{~b}$ shows a trendline comparison for the eight bat shapes, with varying sample size reflecting the different levels of production in the factory through the data collection period (Bat $1=953$, Bat $2=136$, Bat $3=1,471$, Bat $4=885$, Bat $5=747$, Bat $=209$, Bat $7=575$, and Bat $8=29$, Total bats $=5,005)$. All trend lines had $R^{2}$ values $>0.992$. Residuals were checked and showed a clear random pattern indicating that the data was a good fit for a linear model. The trend was close for all eight tested variations in bat profile, which indicates the model was independent of blade shape but dependent on the blade and handle length, and location of CoM.

\section{Discussion}

The two-section one-dimensional beam model to calculate MOI is a simple model of the cricket bat that does not account for the complex depth profile of the blade. Yet this model was shown to estimate MOI with an RMSE of $0.0017 \mathrm{~kg} \mathrm{~m}^{2}$, which equates to $0.43-0.53 \%$ on the MOI range of $0.32-0.39 \mathrm{~kg} \mathrm{~m}^{2}$. The RMSE error was comparable to that reported by Allen [17] and Taraborelli [2] for tennis rackets, but was lower on the percentage error of the measured values since the typical cricket bat MOI is about ten times higher. Taking account of the likely experimental error in the pendulum method, which has been shown to be $<2 \%$, the model MOI error can be expected to be $<3 \%$. This model consistently underestimated MOI when applied to 26 bats measured experimentally (79\% of data). Small errors in the manual measurement of bat dimensions are unlikely to be the source, as rounding to the nearest mm was checked and the calculated MOI was found to be insensitive to rounding. Testing for $\mathrm{a} \pm 2 \%$ variance of measured MOI due to estimated pendulum method error did not change the overall trend for the model to underestimate. Therefore, this can be assumed to be an artefact of the simple beam model approach.

The high degree of correlation obtained between first mass moment and MOI indicates that bat mass and position of CoM are the dominant parameters that contribute to the inertial properties of the bat. This has already been shown in baseball [9], and in tennis where Taraborelli used a stepwise linear regression model to demonstrate this finding [2]. For cricket bats the two-section beam model does not appear to be sensitive to neglecting the bat depth profile. This is perhaps counter-intuitive on observation but illustrates the significance that centre of mass location has on MOI. First moment also becomes an option for estimating MOI but requires a calibration with $\mathrm{MOI}$ to establish the relationship

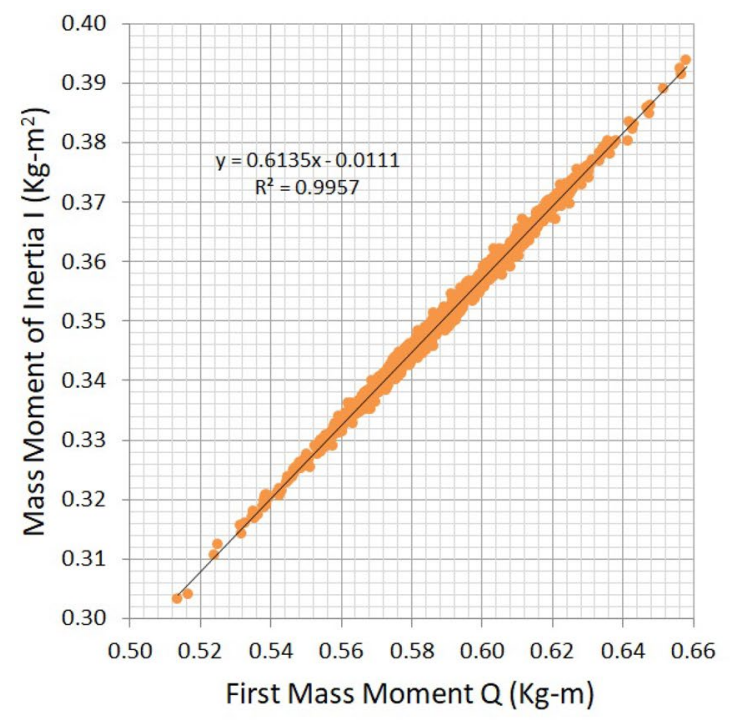

(a)

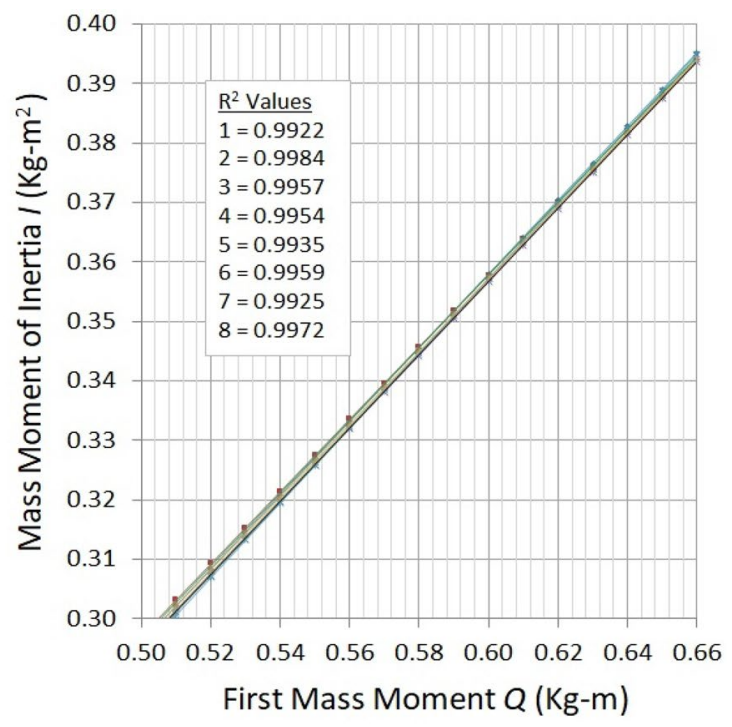

(b)

Fig. 4 First moment vs estimated MOI for a Bat shape 3, b trendline comparison for all eight bat shapes

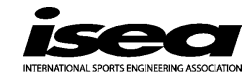


for each shape of bat. This is practical for a bat maker, as once the relationship has been derived (the equivalent bestfit line equation shown in Fig. 4a) they only need to measure mass and location of centre of mass for each bat produced, if handle and blade lengths are kept constant. The proxy is less applicable for one-off measurements by an individual, where it is best to use the two-section beam model. Bat makers could provide a simple web application on their website to make the model calculation based on bat parameters entered by the individual.

In measuring the dimensions of handle and blade length, the location of the bat handle-blade datum was an empirical construct and could be a source of error. However, a sensitivity analysis showed that MOI was insensitive to a $\pm 2 \mathrm{~mm}$ movement of this datum. Human error of marking the datum is likely to be less than this tolerance. Observing that the handle and blade lengths of the measured bats were similar, a test was made on whether a constant blade-handle ratio could simplify the model. When a mean value for the blade-handle ratio (1.86:1) was used from the measured bats in this study the error on MOI rose to $6 \%$. In addition, the simplified model only eliminates one measurement of either handle or blade length depending on which is substituted. Where a bat maker has a production process that makes blades and handles at the same length for a specific size bat, then only one initial measurement is needed for the model, and simplification is unnecessary.

The estimated error from the two-section beam model in this study is unlikely to be large enough to be detectable by any player. Indeed, studies in tennis for MOI [19] and golf for first moment [22] showed the limit of player sensitivity to be under $3 \%$. Assuming a similar sensitivity for cricket bats this provides confidence that the model error is acceptable. However, further work to test sensitivity to MOI in cricket would be the next step to validate the model further.

\section{Conclusions}

The study demonstrated that MOI for cricket bats estimated from a two-section one-dimensional beam model was shown to have an error of $0.43-0.53 \%$ on experimentally measured values. The study has also shown the more easily measured first moment to be well correlated with estimated MOI from the two-section beam model. Therefore, first moment can act as an acceptable proxy measure for MOI, and provides greater simplicity in bat measurements, which some may prefer. Overall, players and bat makers can now easily estimate MOI and thus reduce subjectivity in bat selection.

Supplementary Information The online version contains supplementary material available at https://doi.org/10.1007/s12283-021-00349-y.
Acknowledgements The authors wish to thank Unicorn Products Ltd for their support and cooperation in the design, production and operation of the device to collect first moment data in their factory.

Funding Unicorn Products Ltd supported the design and production of the first moment measurement device.

\section{Declarations}

Conflict of interest The authors declare that they have no conflict of interest.

Open Access This article is licensed under a Creative Commons Attribution 4.0 International License, which permits use, sharing, adaptation, distribution and reproduction in any medium or format, as long as you give appropriate credit to the original author(s) and the source, provide a link to the Creative Commons licence, and indicate if changes were made. The images or other third party material in this article are included in the article's Creative Commons licence, unless indicated otherwise in a credit line to the material. If material is not included in the article's Creative Commons licence and your intended use is not permitted by statutory regulation or exceeds the permitted use, you will need to obtain permission directly from the copyright holder. To view a copy of this licence, visit http://creativecommons.org/licenses/by/4.0/.

\section{References}

1. Brody H (1985) The moment of inertia of a tennis racket. Phys Teach 23(4):213-216

2. Taraborrelli L, Grant R, Sullivan M, Choppin S, Spurr J, Haake $S$, Allen T (2019) Recommendations for estimating the moments of inertia of a tennis racket. Sports Eng. https://doi.org/10.1007/ s12283-019-0303-8

3. Eftaxiopoulou T, Persad L, Bull AM (2016) Assessment of performance parameters of a series of five 'historical' cricket bat designs. Proc IMechE Part P J Sports Eng Technol 231(1):57-62

4. Headrick J, Renshaw I, Pinder RA, Davids K (2012) Attunement to haptic information helps skilled performers select implements for striking a ball in cricket. Atten Percept Psychophys 74(8):1782-1791

5. Cross R, Bower R (2006) Effects of swing-weight on swing speed and racket power. J Sports Sci 24(1):23-30

6. Bahill A (2004) The ideal moment of inertia for a baseball or softball bat. IEEE Trans Syst Man Cybern Part A Syst Hum 34(2):197-204

7. Beak S, Davids K, Bennett S (2000) One size fits all? Sensitivity to moment of inertia information from tennis rackets in children and adults. In: Haake S, Coe A (eds) Tennis science and technology. Blackwell Science, Oxford, pp 109-118

8. Fleisig GS, Zheng N, Stodden DF, Andrews JR (2002) Relationship between bat mass properties and bat velocity. Sports Eng $5(1): 1-8$

9. Cross R, Nathan AM (2009) Performance versus moment of inertia of sporting implements. Sports Technol 2(1-2):7-15

10. Schorah D, Choppin S, James D (2012) Investigating the relationship between swing weight and swing speed across different sports using historical data. Proced Eng 34:766-771

11. Stretch RA, Bartlett R, Davids K (2000) A review of batting in men's cricket. J Sports Sci 18(12):931-949

12. Peploe C, McErlain-Naylor SA, Harland AR, King MA (2019) Relationships between technique and bat speed, post-impact ball 
speed, and carry distance during a range hitting task in cricket. Hum Mov Sci 63:34-44

13. Koenig K, Mitchell ND, Hannigan TE, Clutter JK (2004) The influence of moment of inertia on baseball/softball bat swing speed. Sports Eng 7(2):105-117

14. ASTM International (2020) Standard test method for measuring moment of inertia and center of percussion of a baseball or softball bat (ASTM F2398-11(2020)), West Conshohocken, PA. http://www.astm.org

15. Cross R (2001) Customising a tennis racket by adding weights. Sports Eng 4(1):1-14

16. Goodwill S (2002) The Dynamics of tennis ball impacts on tennis rackets. PhD thesis, University of Sheffield, UK. Retrieved from http://etheses.whiterose.ac.uk/12877/

17. Allen T, Grant R, Sullivan M, Taraborrelli L (2018) Recommendations for measuring tennis racket parameters. Proceedings (Brisbane) 2(6):263

18. Kreifeldt JG, Chuang M (1979) Moment of inertia: psychophysical study of an overlooked sensation. Science 206(2):588-590

19. Brody H (2000) Player sensitivity to the moments of inertia of a tennis racket. Sports Eng 3(2):145-148
20. Maltby R (1995) Golf club design, fitting, alteration and repair: the principles and procedures, revised edition. Ralph Maltby Enterprises, Newark

21. Seltzer LZ (2007) Golf: the science and the art, mustang. Tate Publishing \& Enterprises, Oklahoma

22. Harper TE, Roberts JR, Jones R (2005) Driver swingweighting: a worthwhile process. Proc Inst Mech Eng Part B J Eng Manuf 219(5):385-393

23. Spurr J, Goodwill S, Kelley J, Haake S (2014) Measuring the inertial properties of a tennis racket. Proced Eng 72:569-574

24. Eftaxiopoulou T, Narayanan A, Dear JP, Bull AMJ (2011) A performance comparison between cricket bat designs. Proc IMechE Part P J Sports Eng Technol 226(1):16-23

25. Schorah D, Choppin S, James D (2014) Effect of moment of inertia and physical profile on restricted motion swing speed. Proced Eng 72:593-598

Publisher's Note Springer Nature remains neutral with regard to jurisdictional claims in published maps and institutional affiliations. 